\title{
A Review on the Application of RNA based Nanotechnology in In Vivo Computation
}

Harishchander Anandaram*

Department of Bioinformatics, Sathyabama University, Chennai, Tamilnadu, India

*Corresponding author: Harishchander Anandaram, Department of Bioinformatics, Sathyabama University, Chennai, Tamilnadu, India, Tel: +374 91 474 169; E-mail: harishchander.a@gmail.com

Received date: December 15, 2017; Accepted date: February 1, 2018; Published date: February 8, 2018

Copyright: () 2018 Anandaram H. This is an open-access article distributed under the terms of the Creative Commons Attribution License, which permits unrestricted use, distribution, and reproduction in any medium, provided the original author and source are credited.

\begin{abstract}
Computing in molecular-scale has been explored from the era of pre genomics with a limitation in Moore's law for devices based on silicon. The development of in vivo computation requires low energy consumption in molecularscale. The concept of DNA computing has rationalized the basic Boolean functions into multi-layered circuits. In the Recent year, the concept of RNA nanotechnology has emerged as an alternative approach because the RNA nanoparticles are emerging as a promising medium for a nanodevice with a platform for computing in molecularscale. In RNA computing, the varieties of small RNA sequences can work antagonistically to carry out computational logic in circuits to execute the process of in vivo computation. Though RNA computation is promising in most cases, but it is still in the infant stage and there are many challenges to collaborations between the people in RNA nanotechnology and computer science to carry out the necessary advancement in performing in vivo computation by RNA Nanotechnology.
\end{abstract}

Keywords: Moore's law; DNA computing; RNA nanotechnology and in vivo computation

\section{Introduction}

In case of embedded systems and silicon-based computers, circuits with nano chip had become a vital aspect in our life [1] and there is a larger requirement for the development of nano processors to develop a proper protocol for performing computation in various levels of communication. In order to solve the large requirement of processor development, the concept of "molecular scale computing" was evolved. In the process of molecular computation, bottom-up approaches were applied to create the biological and chemical layouts to develop computers in a nanoscale level [2-6]. Apart from the process of computation, the central processing systems with an integrated circuit comprises the implication of logic gates to perform the operations of Boolean logic by receiving the true (i.e., 1 for high voltage) and false (i.e., 0 foe low voltage) values resulting in output. The frameworks of Boolean operations were used as a measurement for the identification of interactions and classification under the abele and disabled conditions were represented to analyze the diseased conditions $[7,8]$. At present, most of the Boolean logic gates were operated by a complex and inconvenient method called fluorescence detection [9-14]. As an alternative, a homogeneous detection method by colorimetric technique was also employed by coefficients with a high level of extinction. However, there are more limitations in the application of DNA-based computation in molecular scale computing and the most obvious condition is the intolerable level of execution time in performing the "AND" or "OR" logic because the common execution time is $1-2 \mathrm{~h}$ but the application of three-layer see-saw circuits in DNA based molecular scale computing require more than $6-10 \mathrm{~h}$ to reach a stable state to achieve the final value. The second challenge of DNAbased computation is the limitation of functional proteins required for catalyzing the reactions but engineers and scientists have started to develop advanced techniques in devices with molecular-scale computing to build computers for the next generation such as Neurologic quantum computers and still the approach of DNA-based computation is promising and it will produce good results in future after developments.

RNA nanotechnology is a relatively modern and recent field of science with the application of bottom-up or top-down approaches to build the artificial architectures of RNA in nanoscale. RNA nanotechnology involves the characterization of the physical, chemical and biological properties of artificial RNA scaffolds in computing devices. The concept of "RNA nanotechnology" began in the beginning of post genomics i.e., the late 1990s with the pioneering research of $\mathrm{Dr}$ Peixuan Guo and the findings were published in Zhang et al. [15-18]. In 2004, the same group reported the systematic formation of the pRNA nanoparticles by the technology of palindrome sequencemediated self-annealing [19-22]. In the following years, they were successful in conjugating the pRNA molecules with the therapeutic functionalities such as microRNA and ribozymes [23-28]. These findings initiated the path for the development of RNA nanotechnology. At present, RNA nanotechnology has become a rapidly developing branch in science with a strong implication in diverse fields such as chemistry, microbiology and nanomedicine. Modern perspectives in the applications of the RNA nanotechnology has lead to a more sophisticated era known as the "era of RNA computing".

The vital significance of RNA Nanotechnology relies on the application of RNA biopolymers in the atomic level. In case of RNA, the base pairing of nucleotides can be canonical as well as non canonical and the tertiary interactions of RNA mediate multi-way junctions, bulges and internal hairpin loops. As an additive feature, the versatility of RNA folding is relatively low in energy and this feature is a significant advantage over DNA with respect to the thermodynamic stability [29-31]. Apart from the additive feature, RNA is currently regarded as a subdivision of nucleic acid nanotechnology because of the discovery of the diverse functions of RNA in riboswitch and 
ribozyme [32,33]. It was also suggested that the RNA molecule has an immense potential in the area of intracellular computation.

\section{Application of RNA Nanotechnogy in Designing Computers}

Till date, the concept of RNA nanotechnology has shown the capability to provide a path to form designed complex structures with a précised control in a nanoscale. The field is still in the initial stage to verify the in-depth application in the design of logic gates as a building block for the construction of neurocomputers with RNA nanotechnology but the emphasis is on some of the vital aspects of why it is critical role in the process of catalysis in cell signaling and sensing functions. Unlike the traditional computers RNAs use the concentrations of specific chemical species as signals to implement the functions of logic to build up the network of multiple layer Boolean networks with logic gates AND/OR/NOT [34-37]. The design and synthesis of basic functional circuits are the fundamental tasks for developing a fully functional computing device. Prior to engineering, it is vital to analyze the statistical and molecular aspects of higher order genetic networks to perform complex functions in the nanotechnology-based devices and these devices can exhibit the logic operations (AND, NOR, NAND or OR gates) with filtering of signal.

\section{Application of RNA Nanotechnology in In vivo Computation}

Recent advances in the area of RNA-based therapeutics have broadened the scope of identifying the therapeutic targets for a variety of human diseases ranging from genetic disorders to opportunistic infections. The application of nanotechnology in living systems has already been proven but the evolution of molecular computers was found to be able to operate and communicate directly in a biological environment [6,14]. Rinaudo et al. [38] programmed a device with biomolecular computing to work inside a living cell. They had inserted a plasmid with a specifically encoded DNA and used an external program to control the computation inside the host cell. The mRNA was used to encode a fluorescent protein and the target sequences for small interfering RNA (siRNA). They found that siRNA was able to control the level of fluorescence exhibited by the cell because of the degradation of mRNA. Win and Smolke [37] developed the technique of in vivo programming with an independent computation in the machinery of cell cycle; in their experiment they observed a response to both endogenous and exogenous molecular signals. In their work, a combination of ribozymes and RNA aptamers were used $[39,40]$. The idea behind the work was to utilize the cleaving ability of ribozyme to a specific molecule. They have also demonstrated the operations of Boolean logic using the concentrations of two proteins as input and output, implemented by studying the mechanism of ribozymeaptamer molecules using yeast.

Auslander et al. [41] utilized the advancements in the synthetic biology of RNA and designed the standardized control devices using a trigger with a set of controlled transcription factors and RNA-binding proteins. These combinatorial circuits can be integrated to perform digital computations with NAND, AND, NOT and N-IMPLY expression logic in single mammalian cells. Importantly, they showed that the individual mammalian cells were capable of executing the basic arithmetic functions in a robust manner. As an outcome, this information will provide a new strategy for treatment in future. Lou et al. [42] developed a memory module which is a toggle switch with two mutually repressed repressors (CI and CI434 genes) isolated from the lambda and 434 phages respectively. The memory module is expected to function as follows: when CI is present, it activates transcription and represses the transcription of CI434, thus establishing a stable state of high CI and low CI434 and it is defined as the "ON" state of the memory module. In this case, the red fluorescent protein is expressed. Alternatively, when CI434 is present, it can repress the transcription of $\mathrm{CI}$ and thus maintain the transcription of itself and establishing a stable state of low CI and high CI434 and it is defined as the "OFF" state. Another approach used a scalable factor (transcriptional/posttranscriptional) to construct a synthetic regulatory circuit with a HeLa cancer cell 'classifier' to senses the expression levels of a customizable set of endogenous microRNAs to trigger a cellular response that matches the predetermined profile of interest [43].

\section{Conclusion}

Natural or synthetic RNA molecules can be fold into predefined structures for their spontaneous assembly to form nanoparticles with multiple functionalities. The field of RNA nanotechnology is still emerging but it will play a vital role in synthetic biology medicine, biotechnology and nanotechnology.

\section{Future Prospective}

RNA-based computation has more advantages in terms of variations and flexibility than the DNA-based computation. But, the biochemical reaction time is still a critical aspect in working with a scalable circuit.

\section{Further Challenges}

The biggest challenge associated with the RNA-based computation of intracellular machinery is its instability. However, the artificial modification of the RNA bases, phosphate backbones and the C-2 functional group produces an acceptable stability. Moreover, it is believed that the most prominent replacement of the 2-hydroxyl group with fluorine may have a minimal detrimental effect on RNA folding.

The next challenge is to analyze the issues involved in the instability of in vivo computation by RNA with the operations in logic for a large number of inputs involved in targeting an unexpected pathway because the results RNA folding and in vivo requires further studies to explore the field of "in vivo RNA computation".

The final challenge associated with "in vivo RNA computation" involves the cost for yield and production of RNA because the synthesis of RNA will gradually decrease with the development of technologies for the industrial production of RNA in large scale.

\section{References}

1. Qiu M, Sha EHM (2009) Cost minimization while satisfying hard or soft timing constraints for heterogeneous embedded systems. ACM Trans Des Autom Electron Syst 14: 25.

2. Ogihara M, Ray A (2000) Molecular computation: DNA computing on a chip. Nature 403: 143-144.

3. Benenson Y, Paz-Elizur T, Adar R, Keinan E, Livneh Z (2001) Programmable and autonomous computing machine made of biomolecules. Nature 414: 430-434.

4. Reif JH (2002) Computing: Successes and challenges. Science 296: 478-479. 
5. Benenson Y, Gil B, Ben-Dor U, Adar R, Shapiro E, et al. (2004) An autonomous molecular computer for logical control of gene expression. Nature 429: 423-429.

6. De Silva AP (2005) Molecular computation: molecular logic gets loaded. Nat Mater 4: 15-16.

7. Nagaraj SH, Reverter A (2011) A Boolean-based systems biology approach to predict novel genes associated with cancer: Application to colorectal cancer. BMC Syst Biol 5: 35.

8. Mukherjee S, Pelech S, Neve RM, Kuo WL, Ziyad S, et al. (2009) Sparse combinatorial inference with an application in cancer biology. Bioinformatics 25: 265-271.

9. Seelig G, Soloveichik D, Zhang DY, Winfree E (2006) Enzyme-free nucleic acid logic circuits. Science 314: 1585-1588.

10. Mao C, LaBean TH, Relf JH, Seeman NC (2000) Logical computation using algorithmic selfassembly of DNA triple-crossover molecules. Nature 407: 493-496.

11. Elbaz J, Moshe M,Willner I (2009) Coherent activation of DNA tweezers: A ‘SET-RESET' logic system. Angew Chem Int Ed Engl 48: 3834-3837.

12. Freeman R, Finder T, Willner I (2009) Multiplexed analysis of $\mathrm{Hg} 2+$ and $\mathrm{Ag}+$ ions by nucleic acid functionalized $\mathrm{CdSe} / \mathrm{ZnS}$ quantum dots and their use for logic gate operations. Angew Chem Int Ed Engl 48: 7818-7821.

13. Frezza BM, Cockroft SL, Ghadiri MR (2007) Modular multi-level circuits from immobilized DNA-based logic gates. J Am Chem Soc 129: 14875-14879.

14. Miyoshi D, Inoue M, Sugimoto N (2006) DNA logic gates based on structural polymorphism of telomere DNA molecules responding to chemical input signals. Angew Chem Int Ed Engl 45: 7716-7719.

15. Shu D, Moll WD, Deng Z, Mao C, Guo P (2004) Bottom-up assembly of RNA arrays and superstructures as potential parts in nanotechnology. Nano Lett 4: 1717-1723.

16. Shu Y, Cinier M, Shu D, Guo P (2011) Assembly of multifunctional $\varphi 29$ pRNA nanoparticles for specific delivery of siRNA and other therapeutics to targeted cells. Methods 54: 204-214.

17. Zhang F, Lemieux S,Wu X, St.-Arnaud S, McMurray CT, et al. (1998) Function of hexameric RNA in packaging of bacteriophage $\varphi 29$ DNA in vitro. Mol Cell 2: 141-147.

18. Hendrix RW (1998) Bacteriophage DNA packaging: RNA gears in a DNA transport machine (minireview). Cell 94: 147-150.

19. Shu D, Shu Y, Haque F, Abdelmawla S, Guo P (2011) Thermodynamically stable RNA threeway junctions for constructing multifuntional nanoparticles for delivery of therapeutics. Nat Nanotechnol 6: 658-667.

20. Haque F, Shu D, Shu Y, Shlyakhtenko L, Rychahou P, et al. (2012) Ultrastable synergistic tetravalent RNA nanoparticles for targeting to cancers. Nano Today 7: 245-257.

21. Khaled A, Guo S, Li F, Guo P (2005) Controllable self-assembly of nanoparticles for specific delivery of multiple therapeutic molecules to cancer cells using RNA nanotechnology. Nano Lett 5: 1797-1808.

22. Guo S, Huang F, Guo P (2006) Construction of folate-conjugated pRNA of bacteriophage $\varphi 29$ DNA packaging motor for delivery of chimeric siRNA to nasopharyngeal carcinoma cells. Gene Ther 13: 814-820.

23. Hoeprich S, Zhou Q, Guo S, Qi G, Wang Y, et al. (2003) Bacterial virus $\varphi 29$ pRNA as a hammerhead ribozyme escort to destroy hepatitis B virus. Gene Ther 10: 1258-1267.
24. Shu Y, Cinier M, Fox SR, Ben-Johnathan N, Guo P (2011) Assembly of therapeutic pRNA-siRNA nanoparticles using bipartite approach. Mol Ther 19: 1304-1311.

25. Shu Y, Shu D, Diao Z, Shen G, Guo P (2009) Fabrication of polyvalent therapeutic RNA nanoparticles for specific delivery of siRNA, ribozyme and drugs to targeted cells for cancer therapy. In Proc Life Science Systems and Applications Workshop (LiSSA 2009), Bethesda, Piscataway, NJ: IEEE 2: 9-12.

26. Abdelmawla S, Guo S, Zhang L, Pulukuri S, Patankar P, et al. (2011) Pharmacological characterization of chemically synthesized monomeric pRNA nanoparticles for systemic delivery. Mol Ther 19: 1312-1322.

27. Ye X, Hemida M, Zhang HM, Hanson P, Ye Q, et al. (2012) Current advances in $\varphi 29$ pRNA biology and its application in drug delivery. Wiley Interdiscip Rev RNA 3: 469-481.

28. Ye X, Liu Z, Hemida MG, Yang D (2011) Targeted delivery of mutant tolerant anticoxsackievirus artificial microRNAs using folate conjugated bacteriophage $\varphi 29$ pRNA. PLoS ONE 6: e21215.

29. Searle MS, Williams DH (1993) On the stability of nucleic acid structures in solution: enthalpy-entropy compensations, internal rotations and reversibility. Nucleic Acids Res 21: 2051-2056.

30. Sugimoto N, Nakano S, Katoh M, Matsumura A, Nakamuta H, et al. (1995) Thermodynamic parameters to predict stability of RNA/DNA hybrid duplexes. Biochemistry 34: 211-216.

31. Freier SM, Kierzek R, Jaeger JA, Sugimoto N, Caruthers MH, et al. (1986) Improved free-energy parameters for predictions of RNA duplex stability. Proc Natl Acad Sci 83: 9373-9377.

32. Guo P, Erickson S, Anderson D (1987) A small viral RNA is required for in vitro packaging of bacteriophage $\varphi 29$ DNA. Science 236: 690-694.

33. Reif R, Haque F, Guo P (2013) Fluorogenic RNA nanoparticles for monitoring RNA folding and degradation in real time in living cells. Nucleic Acid Ther 22: 428-437.

34. Guo P (2010) The emerging field of RNA nanotechnology. Nat Nanotechnol 5: 833-842.

35. Benenson Y (2009) RNA-based computation in live cells. Curr Opin Biotechnol 20: 471-478.

36. Breaker RR (2008) Complex riboswitches. Science 319: 1795-1797.

37. Win MN, Smolke CD (2008) Higher-order cellular information processing with synthetic RNA devices. Science 322: 456-460.

38. Rinaudo K, Bleris L, Maddamsetti R, Subramanian S,Weiss R, et al. (2007) A universal RNAi-based logic evaluator that operates in mammalian cells. Nat Biotechnol 25: 795-801.

39. Breaker RR (2002) Engineered allosteric ribozymes as biosensor components. Curr Opin Biotechnol 13: 31-39.

40. Suess B, Weigand JE (2008) Engineered riboswitches: overview, problems and trends. RNA Biol 5: 24-29.

41. Auslander S, Auslander D, Muller M, Wieland M, Fussenegger M (2012) Programmable single-cell mammalian biocomputers. Nature 487: 123-127.

42. Lou C, Liu X, Ni M, Huang Y, Huang Q, et al. (2010) Synthesizing a novel genetic sequential logic circuit: a push-on push-off switch. Mol Syst Biol 6: 350 .

43. Xie Z, Wroblewska L, Prochazka L, Weiss R, Benenson Y (2011) Multiinput RNAi-based logic circuit for identification of specific cancer cells. Science 333: 1307-1311. 